\title{
Article \\ A Framework for Crowd Management during COVID-19 with Artificial Intelligence
}

\author{
Mishaal M. Almutairi ${ }^{1}$, Mohammad Yamin ${ }^{2, *} \mathbb{D}$, George Halikias ${ }^{1}$ and Adnan Ahmed Abi Sen ${ }^{3}$ \\ 1 Department of Electrical and Electronic Engineering, School of Mathematics, Computer Science and \\ Engineering, London EC1V 0HB, UK; Mishaal.Almutairi@city.ac.uk (M.M.A.); g.halikias@city.ac.uk (G.H.) \\ 2 Faculty of Economics and Administration, King Abdulaziz University, Jeddah 21589, Saudi Arabia \\ 3 Deanship of Information Technology, Islamic University, Madinah 42351, Saudi Arabia; \\ adnanmnm@hotmail.com \\ * Correspondence: myamin@knu.edu.asa
}

check for updates

Citation: Almutairi, M.M.; Yamin, M.; Halikias, G.; Abi Sen, A.A. A Framework for Crowd Management during COVID-19 with Artificial Intelligence. Sustainability 2022, 14 , 303. https://doi.org/10.3390/ su14010303

Academic Editor: Amir Mosavi

Received: 30 September 2021

Accepted: 24 December 2021

Published: 28 December 2021

Publisher's Note: MDPI stays neutral with regard to jurisdictional claims in published maps and institutional affiliations.

Copyright: (c) 2021 by the authors. Licensee MDPI, Basel, Switzerland. This article is an open access article distributed under the terms and conditions of the Creative Commons Attribution (CC BY) license (https:/ / creativecommons.org/licenses/by/ $4.0 /)$.

\begin{abstract}
COVID-19 requires crowded events to enforce restrictions, aimed to contain the spread of the virus. However, we have seen numerous events not observing these restrictions, thus becoming super spreader events. In order to contain the spread of a human to human communicable disease, a number of restrictions, including wearing face masks, maintaining social distancing, and adhering to regular cleaning and sanitization, are critical. These restrictions are absolutely essential for crowded events. Some crowded events can take place spontaneously, such as a political rally or a protest march or a funeral procession. Controlling spontaneous crowded events, like a protest march, political rally, celebration after a sporting event, or concert, can be quite difficult, especially during a crisis like the COVID-19 pandemic. In this article, we review some well-known crowded events that have taken place during the ongoing pandemic. Guided by our review, we provide a framework using machine learning to effectively organize crowded events during the ongoing and for future crises. We also provide details of metrics for the validation of some components in the proposed framework, and an extensive algorithm. Finally, we offer explanations of its various functions of the algorithm. The proposed framework can also be adapted in other crises.
\end{abstract}

Keywords: COVID-19; crowded events; framework; machine learning; regression

\section{Introduction}

Organizing crowded events often involve complex tasks, but to organize them during an ongoing crisis or pandemic, such as COVID-19, which requires adherence to many restrictions, is much more difficult. In particular, persuading or forcing participants to maintain adequate social distancing is very difficult. Currently, we are passing through the COVID19 pandemic, a disease caused by a coronavirus [1]. By the middle of November 2021, more than 253,000,000 people had been infected with the virus, and over 5,100,000 lives had been lost. Global efforts to track the spread of the virus has so far received mixed success. Most countries have been experiencing multiple waves of the virus. Since its beginning, several variants of COVID-19 have emerged. The Delta variant of COVID-19, which was first detected in December 2020 in India [2], has caused a large number of deaths in many parts of the globe.

The World Health Organization (WHO) has granted emergency approval for several recently developed vaccines. There has been some hesitancy in the acceptance of vaccines [3], largely due to misinformation or apprehension [4]. Vaccination programs appear to be very successful in many countries, however, the available vaccines have been found to be marginally less effective against the Delta variant [5]. The after-effects of a coronavirus in some people can cause significant problems for a considerable period of time. A study into post-acute COVID-19, a syndrome characterized by persistent symptoms, was conducted by [6]. In order to check the spread of the virus, measures recommended by the 
WHO have been proven to be effective, and are agreed upon globally. These include social distancing, limiting exposure, wearing masks in public places, regularly washing hands, regular sanitization, and so on.

Crowded events, especially social, religious, recreational, and political gatherings, are part of our life. We have witnessed the cancellation or downsizing of many events due to COVID-19. There have also been many out-of-control crowded events with or without the involvement of regulatory bodies (government of the country or the region). Some significant crowded events during the COVID-19 pandemic are reviewed below.

We have witnessed the management of several regular and irregular events during COVID-19. While some of them mandated and implemented pandemic restrictions, others failed to do so. Most of these events are religious in nature and so, the reasons for failure to comply with COVID-19 restrictions may be rooted in religious perceptions, which may or may not be justified. Here, we discuss some important crowded events, and compare their organization before and during the pandemic.

\subsection{The Hajj before and during COVID-19}

Before COVID-19, 2,500,000-3,000,000 pilgrims were permitted to perform Hajj. Before COVID-19, pilgrims from nearly 200 countries would participate in Hajj yearly, which can only be performed through a permit issued by Hajj management months before the event. After the declaration of COVID-19 as a pandemic, all mosques in the KSA, including the grand mosque in Makkah, which houses the Ka'bah, were closed indefinitely. These mosques were opened again in October 2020, but only for those who could comply with the pandemic restrictions. The grand mosque allowed Umrah for 2500 pilgrims in one shift of $3 \mathrm{~h}$. In 2020, the Hajj was permitted to have only 10,000 pilgrims from within the kingdom. These restrictions continued into 2021, but the Hajj was permitted to host 60,000 local pilgrims. Figure 1 shows the Tawaf before and during the pandemic.

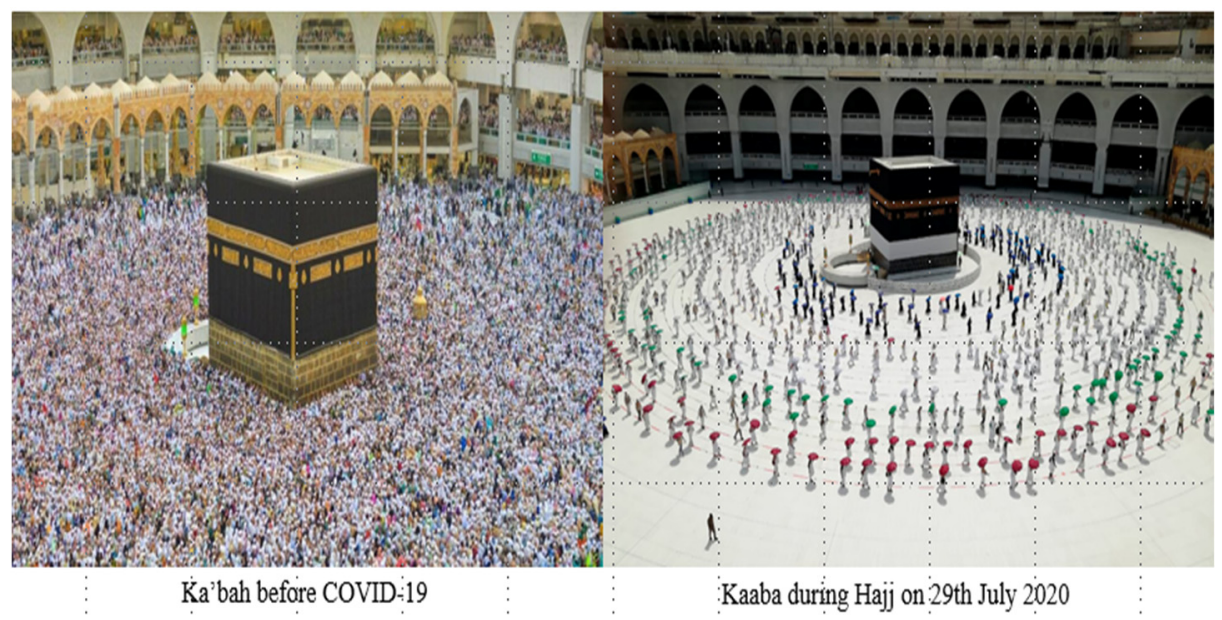

Figure 1. Tawaf before [7] and during [8] the COVID-10 pandemic.

The Umrah was briefly opened to the foreign pilgrims in 2020, but was later closed due to a second wave of the virus, which occurred globally. During the organization of the Hajj, Umrah, and visits to the grand mosque, no serious health issues were reported. With the success of these pilgrimages, the Umrah was again opened to overseas pilgrims from August 2021, and the number of permits has been gradually increased. Permits for pilgrimages are issued online to only those who fulfil COVID-19 requirements, which is a proof of recent vaccination status. For instance, several home-grown apps are provided, which also display the status of the health and vaccination of the pilgrims at the entry level. The Chief of the World Health Organization praised the organization of the Hajj in 2021 [9]. See Figure 2: 


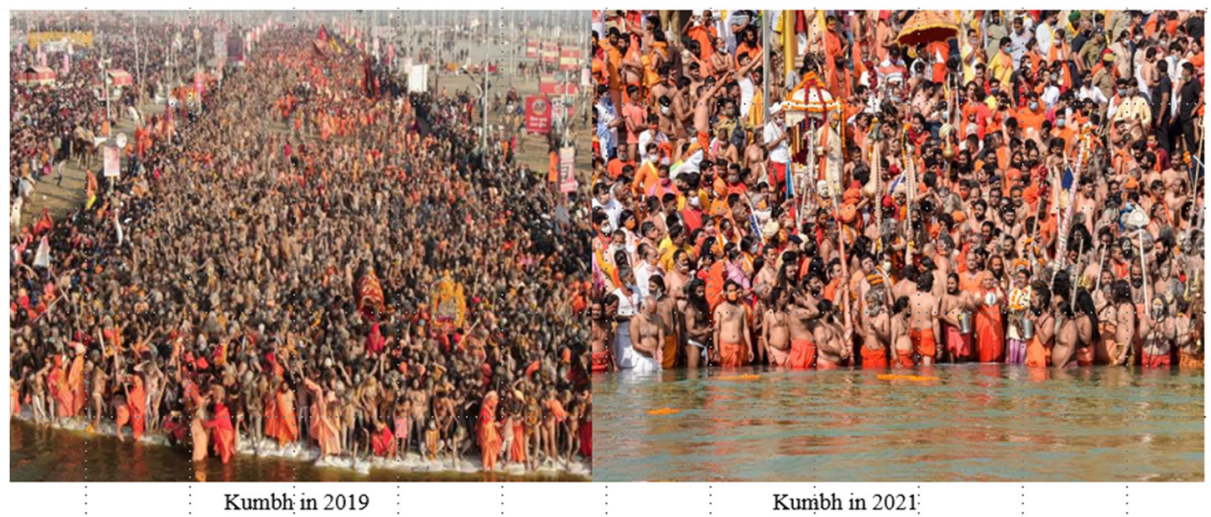

Figure 2. Kumbh in 2019 [10] and 2021 [11].

\subsection{Kumbh before and during COVID-19}

The Kumbh, in 2019, was organized in Prayagraj, previously known as Allahabad, in the province of Utter Pradesh of India. Kumbh, which is a several weeks-long event, is not regulated by a permit and can be attended by anyone. According to [12,13], it was attended by approximately $120,000,000$ pilgrims over a period of about six weeks. In 2021, the Kumbh took place in the city of Haridawar, in the Uttrakhand province of India, in which at least 9,000,000 people participated. Figure 2 shows pilgrims during the 2019 and 2021 events. Unfortunately, COVID-19 restrictions were largely flouted in this event. During this period, India was going through a deadly surge of the Delta variant of COVID-19, which had already killed tens of thousands of people. Many reports, such as [14], suggested that this event served as a super spreader for the ongoing pandemic in India. It is well documented that the Delta variant also spread to dozens of countries, including the USA, UK, France, Germany, Austria, and Australia, and has been proven to be the deadliest of all variants so far.

\subsection{Arbaeen before and during COVID-19}

Arbaeen is an annual pilgrimage to the Karbala in Iraq. It is a pilgrimage of the Shia sect of Islam, which involves a march to the tomb of Hussein (a grandson of the Prophet Mohammad), to mourn his and his family's brutal martyrdom. The pilgrims to this event, apart from Iraq, mainly come from Iran. In normal times, this event would attract several million people each year. Figure 3 shows a snapshot of the 2019 Arbaeen, along with one in 2020. Indeed, the 2020 event was restricted and was only open for local pilgrims, but it still attracted millions of pilgrims and pandemic restrictions were not observed.

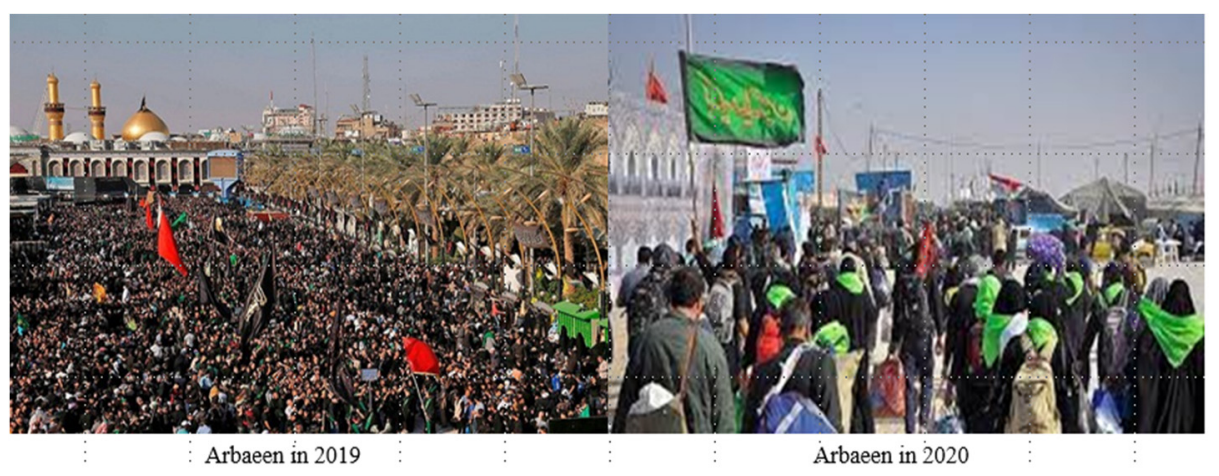

Figure 3. Arbaeen in 2019 [15] and 2021 [16]. 


\subsection{Other Crowded Events during COVID-19}

There have been other crowded events which have flouted all norms and restrictions of COVID-19. One such even was the Semi Final of the 2020 Euro Cup at Wembley Stadium in London. In this event, only vaccinated people were permitted to attend. However, social distancing and face masks were not used by the spectators.

After the match was over, thousands of spectators and supporters took to the streets of London streets, without observing any COVID-19 precautions, as can be seen in Figure 4. It should be noted that this event also took place amidst the ongoing spread of the Delta variant in the UK.
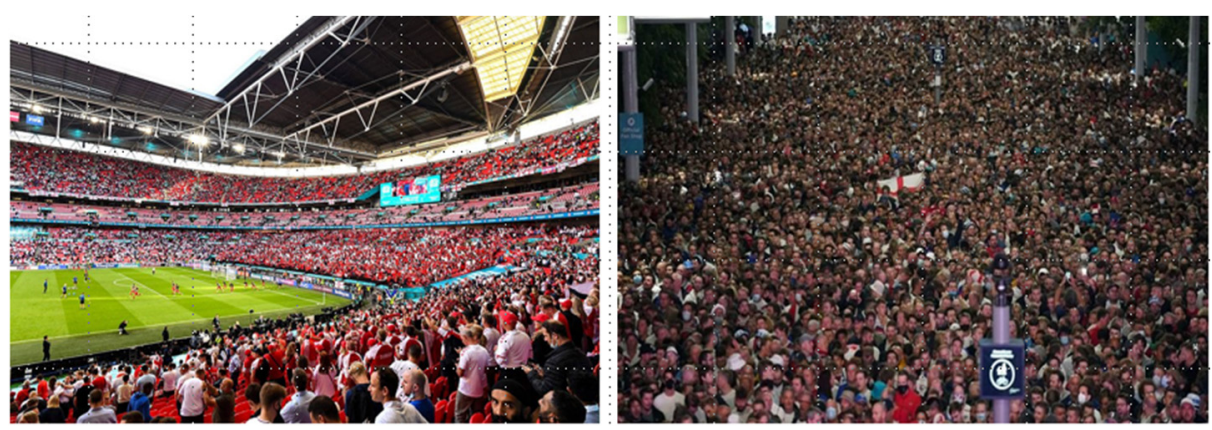

Figure 4. Crowd during [17] and after [18] the 2020 Euro Cup Semi Final in London.

\subsection{Our Contribution in This Article}

In this article, we provide a framework for organizing crowded events during the ongoing pandemic. This framework uses machine learning classification models. The framework is supported with an algorithm. We also provide an explanation of our algorithm. Our design takes into consideration the lessons learned from the preceding analysis of crowded events during the ongoing pandemic. The framework deals with the organization and administration of the event (from proposal to staging), and takes into consideration attributes including the date, time, and area of the location of the event (including the status of the pandemic in the selected area).

\section{Literature Review}

Many researchers have studied crowded events and have contributed towards the improvement of their management. A good account of crowd management can be found in [19-22]. In particular, in [20], the authors present a framework for crowd management, which was implemented in the case of the annual Hajj pilgrimage. In [23], management of crowd levels in interior places or points-of-interest (POI), particularly in the shopping centers or stores, is discussed. In [24], the aspect of social behavior in a crowded event during a pandemic is discussed.

COVID-19 was first identified in December 2019 in China, but it may have been spreading well before that time. Since then, it spread to almost every country. Various strands of the virus have been detected in several countries, and notable variants are Alpha, Beta, Gamma, and Delta, with the last being the most aggressive and dangerous. Many countries have been going through different waves of these strands as well as others. Many vaccines have been developed and approved by the WHO for emergency use. There has been tremendous research [25-30] on different aspects of COVID-19, including different strands of COVID-19 and vaccines.

The Hajj and Umrah [31] are very well-known annual events, for which, in normal times, about 10,000,000 people are permitted to perform these pilgrimages, while tens of millions of people from around the world wait for their turn. The Kumbh Mela (Kumbh) [32] is believed to be the most crowded event, but it only takes place every three years, in four different cities periodically. The Kumbh attracts tens of millions of devotees in the space of five to six weeks. Another crowded event, which also attracts millions of faithful, is 
Arbaeen [33]. There are many other regular, periodic, or irregular events that attract large crowds, an account of which can be found in [19].

\section{Classification vs. Regression}

There are algorithms that can be used for both regression and classification. In the field of machine learning, algorithms are distinguished based on their purpose. If we use machine learning to predict values, we call it regression, and if we use machine learning to choose the category or decide between true and false, we call it classification. Usually, accuracy is used in classification, while in regression we use other metrics, like MSE, RMSE, and MAE. In human thinking, classification can sometimes be fuzzy, which means we may not only have very true or very false, but a value in between the two. It is also possible to fuzzify the binary class membership values. These matters are discussed in detail in the context of neural network algorithms in [34], where it is convincingly asserted that machine learning can "think" between false and true.

In the next section, we discuss the organization of some of these events in normal times and compare them, and their implications, with those organized during the ongoing pandemic, as well as their implications.

\section{A Framework for Crowded Events during a Pandemic}

Many crowded events with a lack of proper management or poor organization that may have contributed to the spread of COVID-19 can be found. We listed a few such events in the previous section. As the pandemic goes on, so do these kinds of events in different parts of the world. The ongoing mismanagement and poor organization has encouraged us to provide a framework for organizing crowded events. Here, we provide details of the proposed framework.

\subsection{Proposed Framework}

Figure 5 depicts a model for the proposed framework for organizing crowded events during the ongoing pandemic. The framework is designed for crowded events during the ongoing COVID-19 pandemic, but can also be used during other pandemics and crises, caused by either natural disasters or manmade blunders. This framework uses knowledge about the prevention of spreading COVID-19 available from the different portals of the World Health Organization. Now, we explain the main modules and functions of the proposed framework.

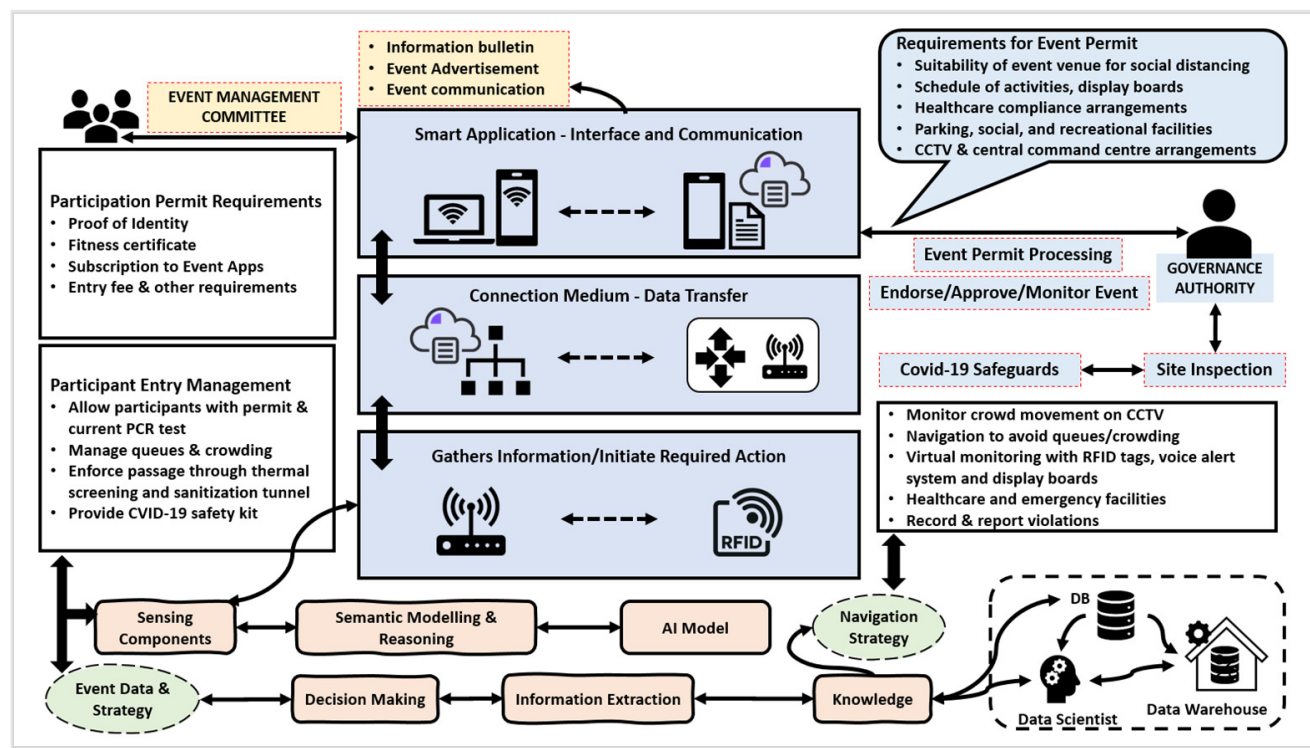

Figure 5. A framework of crowd management during a pandemic. 


\subsubsection{Application for Interface and Communication}

The smart application of the proposed model receives and delivers requests/responses to the users (event management committee and governance authority) of various roles. This layer deploys applications for smart event management to offer various services, including the following: apply for an event permit, endorse/approve/monitor event, guidelines for event/participation, etc.

\subsubsection{Data Transfer for Connection and Networking}

The data transfer module of the proposed model connects and transfers the data for processing. The data are collected from various smart devices through sensors, and this module is responsible for connecting to other devices, servers, etc., over the network. Different devices involved in crowd management through arrangements, security, virtual visits, and inspection, etc., are connected, and data are transferred using this module.

\subsubsection{Information Gathering from Sensing Components}

Various sensors such as temperature sensors, infrared sensors, proximity sensors, and optical sensors, are part of an event management system for detecting and gathering data about the environment. Information is extracted through this module to initiate the necessary action in and around the event timeline.

\subsubsection{Data Management}

The crowd (event attendees) could provide input during the event through various activities and strategies. By means of sensing components or event-based decisions, the data are captured and applied with semantic modelling and reasoning through a machine learning model. The data are stored in a database and will also participate in model fitting, prediction, and forecasting for future actions. In the distributed event environment, all such data origins produce databases and are extracted, transformed, and loaded into the data warehouse. A data scientist makes use of statistical and operational data from the data sources to analyze, predict, and forecast the acquired decisions.

\subsubsection{Semantic Modelling and Reasoning}

Semantic modelling and reasoning do the following:

1. Enrich the meaning of the data being collected from various IoT sensors and actuators.

2. Hide the device heterogeneity and allow for efficient integration.

\subsubsection{Artificial Intelligence Model}

Using semantic web ontologies, this infers a set of new rules based on the prevailing information.

\subsubsection{Knowledge-Driven Decision Making}

An AI model provides knowledge-driven decision-making for cognitive and physical tasks to support event users. This module allows tasks to be accomplished faster and better through better decisions for the event, without human intervention.

Better design and implementation of event strategy and navigation strategy are observed and achieved through AI-based decision making.

\subsection{Algorithm for Proposed Framework}

For the proposed algorithm, classification is more relevant than regression as we only deal with applications requiring accept (true) or reject (false) decisions. In general, one can also choose different machine learning models for an algorithm. A particular algorithm may be better than another (type of data, size of training data, selected features, etc.). On the other hand, some algorithms may have very similar accuracy. In such situations, one may opt to use multiple models with similar accuracy as a committee instead of using the one with the best accuracy. These models can be used to achieve significantly better and 
reliable results. These matters are discussed in [35], where new voting functions for neural network algorithms are discussed.

Here, we provide an algorithm to support the proposed framework. We have chosen two thresholds: Threshold1 is for accepting or rejecting a model based on its accuracy, whereas Threshold 2 is used for checking the number of accepted models in order to ensure reliability. The algorithm uses two functions, one for event planning, and another for event approval (See Algorithm 1).

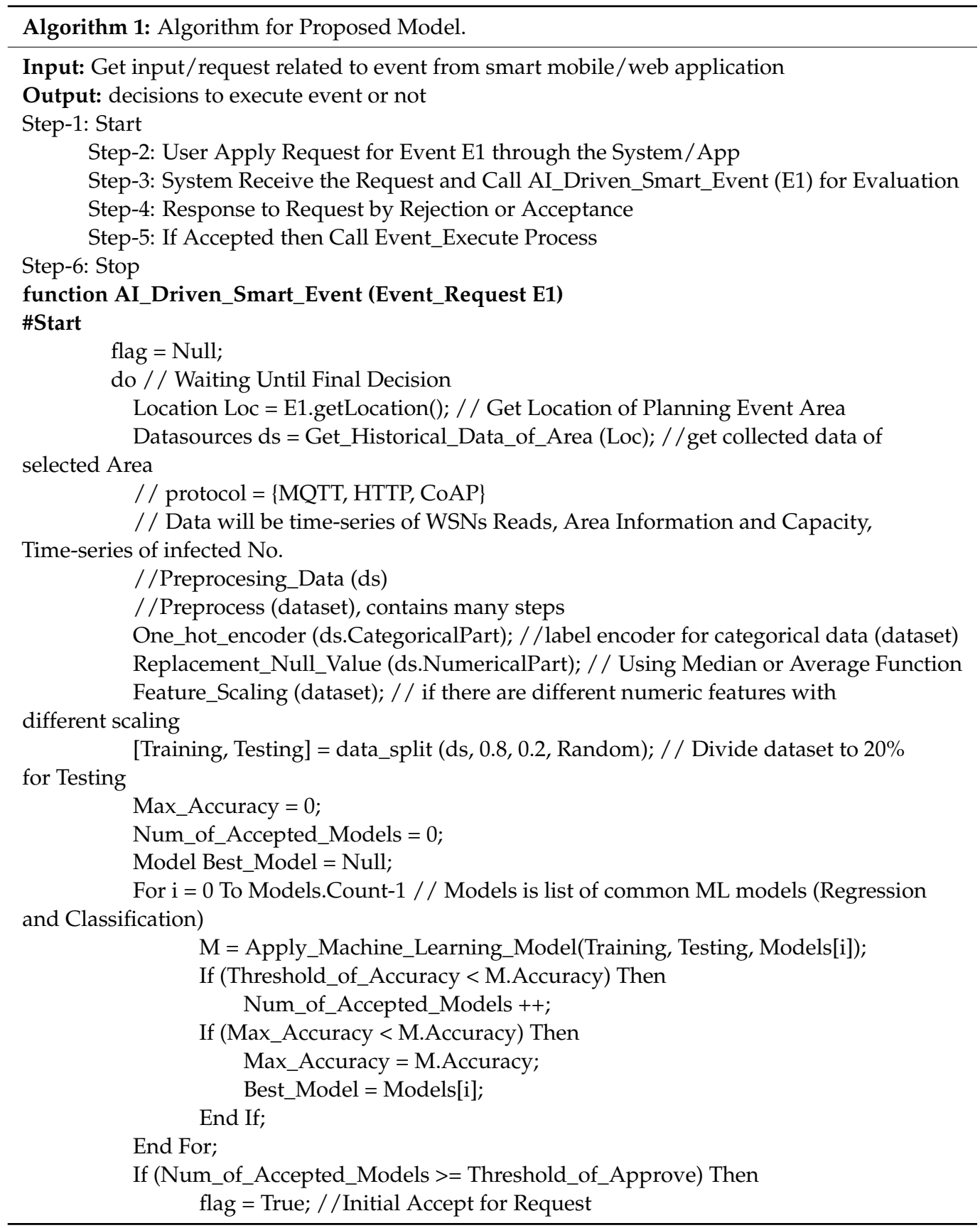




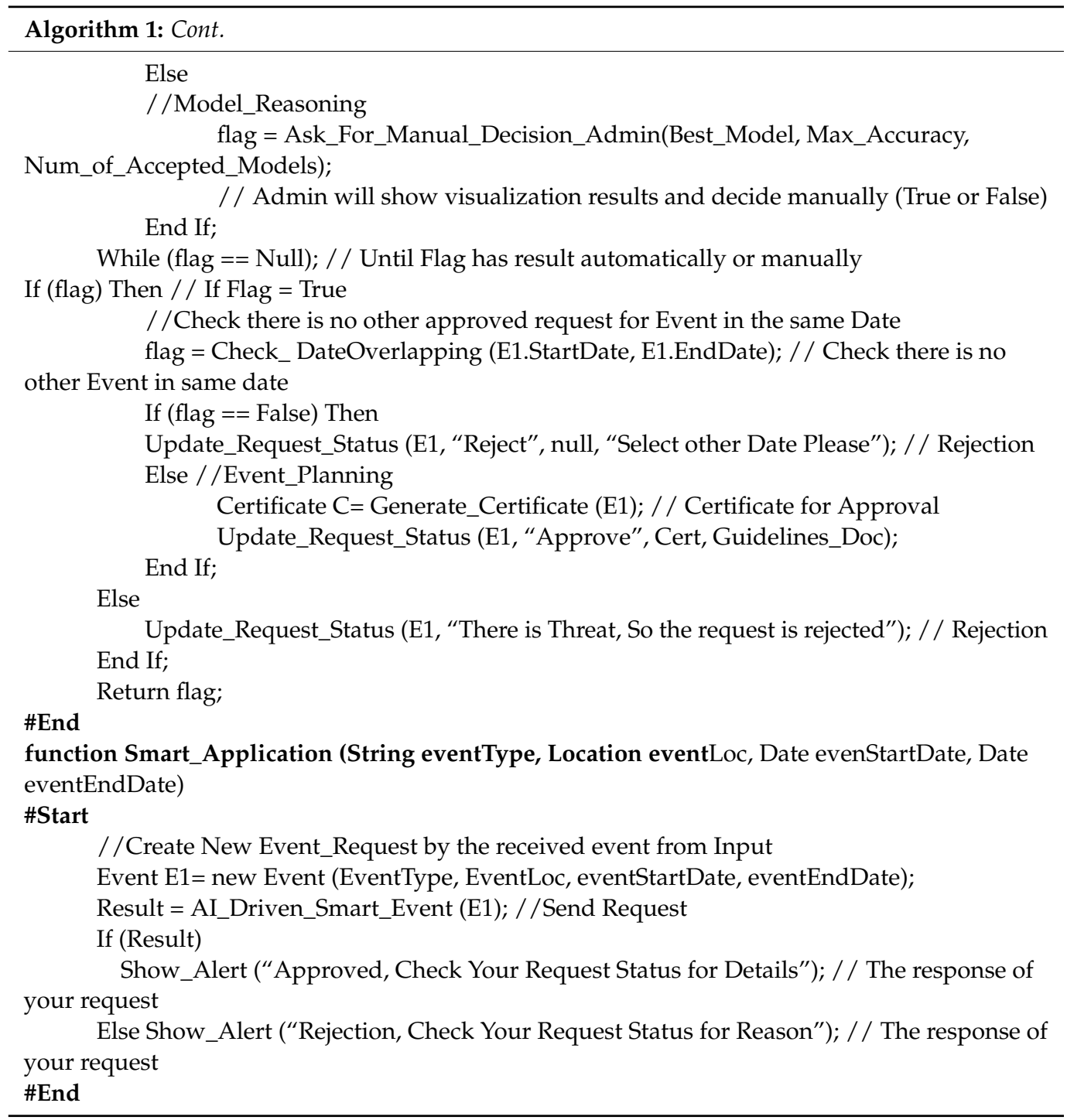

Important Procedures and Policies for Event Execution

Here, we list some procedures and policies to be made available during the execution. Release Info_Bulletin

- Release Event_Advertisement

//event_strategy

- Allow participants with a valid permit and a current PCR test;

- Provide a kit with essential items (i.e., mask, sanitizer, etc.) and a leaflet of information;

- Manage queues by enforcing adequate social distancing;

- Thermal screening, passing through a sanitization tunnel;

- $\quad$ Provide RFID-tagged bracelet for tracking and providing emergency help

//navigation_strategy;

- Monitor crowd movement on CCTV;

- Manage movement to prevent crowding;

- Track individuals with RFID tags as and when required;

- Broadcast alerts through loud speakers and billboards;

- Provide healthcare and emergency support;

- Report violations.

- Collect, store, and manage data from activities and devices (sensors, reports, etc.) for current and future use. 


\subsection{Explanations of Main Functions of the Algorithm}

The algorithm is designed to function in the following manner:

1. The applicant (host/owner/event management committee) would use the Smart Application form to apply for an event permit by inputting the type, location, number of participants, and start and end dates of the event.

2. The Smart Application function will then receive it as a new request and start evaluating the request by invoking the "Smart Event" function, which would process the application through the following steps:

a. Historical data about the selected location (from previous event(s)), which include reports of incidents and infections of previous events for the selected area, would be retrieved. Data from IoT sensors about the pollution and congestion or crowd ratio of the area (time-series) will also be analyzed.

b. Preprocessing of the available data according to its type (numeric or nominal) would take place, which would consist of replacing the null values, scaling numeric values, and encoding the nominal values.

c. The function will then apply common machine learning (ML) models (by going through each of them, one by one), and calculate the accuracy of each step. Note that the function has a list of common ML classification models (managed by the admin), for example, linear regression, log regression, SVM, naive Bayes, decision tree, etc. Thus, the proposed framework depends on different kinds of machine learning models, not just regression but also classification.

d. Then, the data will be divided randomly, with training being $80 \%$ and testing making up the other $20 \%$.

e. The function will calculate the number of models that have an acceptable accuracy (larger than a specific threshold).

f. The function will compare all accuracy values and find the ML model with the maximum value and save this result (to support the admin when managing the list of ML models in the future).

g. The function will check if the number of accepted models is large enough (the threshold can be adjusted by the admin). If so, the function will accept the request automatically when it is verified that there are no threats in this area to host the event.

h. In case the number of accepted models is less than the threshold, the function will request manual reviewing with the accuracy result of the best ML model, in addition to the number of accepted ones.

i. If the return decision is to reject the application, the function will send an alert to the applicant citing safety concerns.

$\mathrm{j}$. If the automatic or manual decision was to approve, the function will check for the availability of the date(s). If the desired date(s) is not available, the function will send a rejection message and advise the applicant to choose another date(s).

k. If the applicant's request is approved, the function will update the status of the request, notify the applicant, send guidelines, generate the certificate of approval, and invoke the Execute Event function (to send an alert to teams to start the process of preparations).

3. It should be noted that we mentioned an important procedure, which contains a number of tasks related to the organization of the event (e.g., alert teams, send ads, monitoring event condition, and feedback). However, all data collected from sensors during the event will automatically be sent to the cloud, as shown in the proposed framework.

4. Feedback and other relevant data will be merged with previous historical data for future evaluation. 


\subsubsection{Factors for Validation for ML Model}

As a result of data not being available, there is no real validation in this work. Instead here, we set out a scheme for future validation of the framework, which can be used to support decisions concerning the management of events during the pandemic situation and other similar scenarios. In other words, our results are simply recommendations, along with some metrics for validation in the future.

The following paragraph provides some common metrics to test ML models in general, which can also be used for the proposed framework, and collect and organize real data (dataset). The dataset can be built manually from the reports of all events and associated applications. Data about COVID-19 from its beginning to up until now would also be useful.

Optimization: Avoiding Model over Fit with an Additional Handout Set

For training in a machine learning model, we usually split data into two parts: $80 \%$ for training and $20 \%$ for testing. The machine learning model provides a data split function to achieve this splitting. After data splitting, the model can do training and testing on these parts, and provides some metrics through which we can verify whether the results are acceptable or not.

\subsubsection{K-Fold Cross Verification}

1. Using classic F-fold cross validation by splitting data into k-folds, $\mathrm{k}=5$;

2. Observations: All combinations and averages of both the test and validation observations;

3. Validated against: Computational complexity and validation accuracy;

4. Optimization: Improving stability of the machine learning model across several data inputs.

This approach splits the given input dataset into K groups (K-fold) of sections of equal sizes. For every learning set, the K-fold prediction function takes $(\mathrm{k}-1)$-folds, and the remaining folds are reserved for the test set. Using this method, the output is observed as less biased when compared with other methods. This method takes a group as the standby or test data set, takes the remaining groups as the training dataset and tries to fit the model on the training set, and assesses the performance of the model using the test set for each group to improve the stability of the model.

\subsubsection{Nested Cross Validation}

1. Using nested cross validation strategy;

2. Observations: Use inner loop (two-fold) for hyper parameter tuning, use outer loop (five-fold) for accuracy estimation;

3. Validated against: Error estimation;

4. Optimization: Improving hyper parameter tuning and estimating accuracy.

The nested cross-validation approach separates the hyper-parameter tuning step and the error estimation step. This process uses the inner loop for hyper-parameter tuning and the outer loop for estimating accuracy.

\subsubsection{Time-Series Cross Validation}

1. Using F-fold $\mathrm{CV}$ by splitting data into k-folds;

2. Observations: Entire training data occurs before your test data;

3. Validated against: Over fitting;

4. Optimization: Improving stability of the machine learning model across several data inputs.

The drawbacks of traditional CV techniques are addressed by using this approach through splitting time series data without instigating data outflow, and using a nested CV to get an impartial estimate of the error on an independent test set and CV with datasets 
that hold multiple time series. This approach improves the stability of the machine learning model across several data inputs and avoids overfitting.

\subsubsection{Model Comparison}

1. Using method $5 \times 2 \mathrm{CV}$ paired $t$-test from Scikit-learn Python machine learning library;

2. Observations: Statistically significant;

3. Validated against: $p$-value;

4. Optimization: Choose the best model.

Based on the statistical significance of the machine learning models, the best model that fits the data to provide better predictions is identified. A $5 \times 2 \mathrm{CV}$ paired $t$-test model is used to relate machine learning models based on their strong statistical foundation. This model would split the data into training $(80 \%)$ and testing $(20 \%)$, and select the testing part $(20 \%)$ randomly five times. Let $C_{a}, C_{b}, C_{c}, C_{d}$, and $C_{e}$ be five classifiers, test each classifier on the training data, and compute $\left(\mathrm{Diff}_{\mathrm{i}}\right)$ the difference in the accuracy between these classifiers. In this way, the process goes through five iterations, which can be used to compute the mean variance $\left(\mathbf{S}^{2}\right)$. Then, $\mathrm{t}$-statistic can be computed from the following formula:

$$
\text { t-statistic }=\frac{\operatorname{Diff} A_{1}}{\sqrt{\frac{1}{5}} \sum_{i=1}^{5} \mathbf{S}_{\mathbf{i}}^{2}}
$$

where $\operatorname{Diff} A_{1}$ is the mean variance of the first iteration.

\subsubsection{Future Research}

In future research, we will provide validation of the proposed algorithm using a suitable dataset from the operations of the system. Additionally, we will provide methods to manage and ensure privacy and security in the proposed framework. We will also deal with the interoperability issue for converting heterogeneous to homogeneous data so as to better support different applications.

\section{Conclusions}

The organization and management of crowded events during a crisis, like the one created by the ongoing coronavirus pandemic, are quite different to those in normal circumstances. Large crowds, especially during a crisis, cannot be managed effectively and efficiently without a proper framework equipped with appropriate technologies. A crowd cannot be blamed for human errors, and a lack of resources, infrastructure, training and experience, and technology. Addressing one aspect of crowding would not remarkably solve crowding problems, rather there must be a comprehensive and sustainable solution. This paper has proposed a comprehensive and sustainable solution to the problems encountered by crowd management. Unfortunately, some crowds grow out of manageable control. Even the best strategies and technologies would not work against excessively large and unmanageable crowds. Crowd management would improve if the responsibility for mismanagement is given to the responsible officials. Equally, there should be a reward for good management. Unfortunately, many crowding events have been poorly organized for social and political gains, some of which have acted as super spreaders in the ongoing pandemic. No religious event or sport is above human lives. We believe that the proposed COVID-19 compliant framework can be very helpful to minimize the spread of the coronavirus. This framework can easily be adapted to manage crowds during different kinds of crises.

Author Contributions: Conceptualization, M.M.A. and M.Y.; methodology, M.M.A., M.Y., G.H. and A.A.A.S.; software, M.Y. and A.A.A.S.; validation, M.Y. and A.A.A.S.; formal analysis, M.M.A., M.Y. and G.H.; investigation, M.M.A., M.Y., G.H. and A.A.A.S.; resources, M.M.A. and G.H.; data curation, M.M.A.; writing—original draft preparation, M.M.A. and M.Y.; writing—review and editing, M.M.A. and M.Y.; visualization, M.Y., M.M.A. and A.A.A.S.; supervision, M.Y. and G.H.; project 
administration, M.Y.; funding acquisition, M.M.A. All authors have read and agreed to the published version of the manuscript.

Funding: This research received no external funding.

Conflicts of Interest: The authors declare no conflict of interest.

\section{References}

1. Wu, Y.-C.; Chen, C.-S.; Chan, Y.-J. The outbreak of COVID-19: An overview. J. Chin. Med. Assoc. 2020, 83, 217-220. [CrossRef] [PubMed]

2. Planas, D.; Veyer, D.; Baidaliuk, A.; Staropoli, I.; Guivel-Benhassine, F.; Rajah, M.M.; Planchais, C.; Porrot, F.; Robillard, N.; Puech, J.; et al. Reduced sensitivity of SARS-CoV-2 variant Delta to antibody neutralization. Nature 2021, 596, 276-280. [CrossRef] [PubMed]

3. Solís Arce, J.S.; Warren, S.S.; Meriggi, N.F.; Scacco, A.; McMurry, N.; Voors, M.; Syunyaev, G.; Malik, A.A.; Aboutajdine, S.; Adeojo, O.; et al. COVID-19 vaccine acceptance and hesitancy in low- and middle-income countries. Nat. Med. 2021, 27, 1385-1394. [CrossRef]

4. Loomba, S.; de Figueiredo, A.; Piatek, S.J.; de Graaf, K.; Larson, H.J. Measuring the impact of COVID-19 vaccine misinformation on vaccination intent in the UK and USA. Nat. Hum. Behav. 2021, 5, 337-348, Correction in 2021, 5, 960. [CrossRef]

5. Pouwels, K.B.; Pritchard, E.; Matthews, P.C.; Stoesser, N.; Eyre, D.W.; Vihta, K.-D.; House, T.; Hay, J.; Bell, J.I.; Newton, J.N.; et al. Effect of Delta variant on viral burden and vaccine effectiveness against new SARS-CoV-2 infections in the UK. Nat. Med. 2021, 27, 1-9. [CrossRef] [PubMed]

6. Nalbandian, A.; Sehgal, K.; Gupta, A.; Madhavan, M.V.; McGroder, C.; Stevens, J.S.; Cook, J.R.; Nordvig, A.S.; Shalev, D.; Sehrawat, T.S.; et al. Post-acute COVID-19 syndrome. Nat. Med. 2021, 27, 601-615. [CrossRef]

7. Arora, A. COVID-19: Saudi Authorities Say Only 'immunised' Pilgrims Will Be Allowed in Mecca. Republic World, 2021. Available online: https://www.republicworld.com/world-news/middle-east/covid-19-saudi-authorities-say-only-immunisedpilgrims-will-be-allowed-in-mecca.html (accessed on 30 September 2021).

8. Kalebi, A. Pilgrimage in a Pandemic: Lessons from Mecca on Containing Covid-19. Business Standard, 2021. Available online: https:/ / www.business-standard.com/article/current-affairs/pilgrimage-in-a-pandemic-lessons-from-mecca-on-containingcovid-19-121051500151_1.html (accessed on 30 September 2021).

9. WHO Chief Lauds Saudi Arabia's Coronavirus Measures During Hajj. Saudi Gazette. Available online: https://www.saudigazette. com.sa/article/608957/SAUDI-ARABIA/WHO-chief-lauds-Saudi-Arabias-coronavirus-measures-during-Hajj (accessed on 30 September 2021).

10. Kumbh, M. 2019: At Rs 4,236 Crore, This Year's Mela in Prayagraj is Costliest Ever. Business Today, 2019. Available online: https:/ / www.businesstoday.in/latest/economy-politics / story/kumbh-mela-2019-at-rs-4236-crore-this-year-mela-costliestever-160612-2019-01-16 (accessed on 30 September 2021).

11. Nearly 50 Kumbh Mela Visitors Test Positive for COVID-19 on Returning to Gujarat. India Today, 2021.

12. Kumbh, M. The World's Largest Religious Gathering Sets 3 World Records. Business Insider (India). Available online: https:/ www. businessinsider.in/kumbh-mela-2019-sets-3-guinness-world-record/articleshow /68250313.cms (accessed on 30 September 2021).

13. Govt data. 9.1 Million thronged Mahakumbh Despite COVID-19 Surge. Hindustan Times. Available online: https: / / www.hindustantimes.com/cities/dehradun-news/91-million-thronged-mahakumbh-despite-covid-19-surge-govt-data-10 1619729096750.html (accessed on 30 September 2021).

14. NDTV Coronavirus, April 14, 2021, over 1700 Test COVID-19 Positive at Haridwar Kumbh over 5 Days. Available online: https: / / www.ndtv.com/india-news / over-1-700-test-covid-19-positive-at-haridwar-kumbh-over-5-days-official-2414270 (accessed on 30 September 2021).

15. AFP. Arbaeen: 'If You Want to See Humanity Living and Breathing, Experience This Walk'. The Express Tribune, 2018. Available online: https:/ / tribune.com.pk/article/74031/arbaeen-if-you-want-to-see-humanity-living-and-breathing-experience-thiswalk (accessed on 30 September 2021).

16. Tasnim. No Foreign Pilgrims Allowed in Iraq for Arbaeen. Iraq Business News, 2020. Available online: https://www.iraqbusinessnews.com/2020/09/02/no-foreign-pilgrims-allowed-in-iraq-for-arbaeen/ (accessed on 30 September 2021).

17. GettyImages. Euro 2020: Fans Euphoric as England Beat Denmark to Reach Final. BBC, 2021. Available online: https://www.bbc. $\mathrm{com} /$ news / in-pictures-57743446 (accessed on 30 September 2021).

18. Sarmah, B. Euro 2020 and a Fresh Spike in COVID-19 Cases. Hindustan Times, 2021. Available online: https://www. hindustantimes.com/sports/football/euro-2020-and-a-fresh-spike-in-covid-19-cases-101625844271740.html (accessed on 30 September 2021).

19. Yamin, M.; Ades, Y. Crowd Management with RFID and Wireless Technologies. In Proceedings of the 2009 First International Conference on Networks \& Communications, Chennai, India, 27-29 December 2009; pp. 439-442. [CrossRef]

20. Yamin, M.; Basahel, A.M.; Abi Sen, A.A. Managing Crowds with Wireless and Mobile Technologies. Wirel. Commun. Mob. Comput. 2018, 2018, 1-15. [CrossRef]

21. Felemban, E.; Sheikh, A.A.; Naseer, A. Improving Response Time for Crowd Management in Hajj. Computers 2021, 10, 46. [CrossRef] 
22. Owaidah, A.; Olaru, D.; Bennamoun, M.; Sohel, F.; Khan, N. Review of Modelling and Simulating Crowds at Mass Gathering Events: Hajj as a Case Study. J. Artif. Soc. Soc. Simul. 2019, 22, 1-36. [CrossRef]

23. Polanco, L.D.; Siller, M. Crowd management COVID-19. Annu. Rev. Control. 2021, 52, 465-478. [CrossRef] [PubMed]

24. Templeton, A. Future research avenues to facilitate social connectedness and safe collective behavior at organized crowd events. Group Process. Intergroup Relat. 2021, 24, 216-222. [CrossRef]

25. Bardesi, H.; Al-Mashaikhi, A.; Basahel, A.; Yamin, M. COVID-19 compliant and cost effective teaching model for King Abdulaziz University. Int. J. Inf. Technol. 2021, 13, 1343-1356. [CrossRef]

26. Bajaba, S.; Mandurah, K.; Yamin, M. A framework for pandemic compliant higher education national system. Int. J. Inf. Technol. 2021, 13, 407-414. [CrossRef]

27. Yamin, M.; Abi Sen, A.A.; AlKubaisy, Z.M.; Almarzouki, R. A Novel Technique for Early Detection of COVID-19. Comput. Mater. Contin. 2021, 68, 2283-2298. [CrossRef]

28. Khanday, A.M.U.D.; Rabani, S.T.; Khan, Q.R.; Rouf, N.; Din, M.M.U. Machine learning based approaches for detecting COVID-19 using clinical text data. Int. J. Inf. Technol. 2020, 12, 731-739. [CrossRef]

29. Yadav, R.S. Data analysis of COVID-2019 epidemic using machine learning methods: A case study of India. Int. J. Inf. Technol. 2020, 12, 1321-1330. [CrossRef]

30. Khanday, A.M.U.D.; Khan, Q.R.; Rabani, S.T. Identifying propaganda from online social networks during COVID-19 using machine learning techniques. Int. J. Inf. Technol. 2021, 13, 115-122. [CrossRef] [PubMed]

31. Ministry of Hajj and Umrah. Available online: https:/ / www.haj.gov.sa/en (accessed on 30 September 2021).

32. Yamin, M. Managing crowds with technology: Cases of Hajj and Kumbh Mela. Int. J. Inf. Technol. 2019, 11, 229-237. [CrossRef]

33. Nikjoo, A.; Sharifi-Tehrani, M.; Karoubi, M.; Siyamiyan, A. From Attachment to a Sacred Figure to Loyalty to a Sacred Route: The Walking Pilgrimage of Arbaeen. Religions 2020, 11, 145. [CrossRef]

34. Tajti, T. Fuzzification of training data class membership binary values for neural network algorithms. Annal. Math. Inform. 2020, 52, 217-228. [CrossRef]

35. Tajti, T. New voting functions for neural network algorithms. Annal. Math. Inform. 2020, 52, 229-242. [CrossRef] 